\title{
A New Look for Starlike Logharmonic Mappings
}

\section{Zayid Abdulhadi}

Department of Mathematics, American University of Sharjah, Sharjah, UAE

Email: zahadi@aus.edu

Received 25 November 2013; revised 25 December 2013; accepted 2 January 2014

Copyright (C) 2014 by author and Scientific Research Publishing Inc.

This work is licensed under the Creative Commons Attribution International License (CC BY). http://creativecommons.org/licenses/by/4.0/

(c) (i) Open Access

\section{Abstract}

A function $f(z)$ defined on the unit disc $U$ is said to be logharmonic if it is the solution of the nonlinear elliptic partial differential equation $\frac{\overline{f_{\bar{z}}}}{\bar{f}}=a \frac{f_{z}}{f}$, where $a \in H(U)$ such that $a(U) \subset U$. These mappings admit a global representation of the form $f(z)=z|z|^{2 \beta} h(z) \overline{g(z)}$, where $\mathfrak{R}(\beta)>-1 / 2$. In this paper,we shall consider the logharmonic mappings $f(z)=z h(z) \overline{g(z)}$, where $\phi=z h(z)$ is starlike. Distortion theorem and radius of starlikess are obtained. Moreover, we use star functions to determine the integral means for these mappings. An upper bound for the arclength is included.

\section{Keywords}

Logharmonic, Univalent, Starlike, Integral Means, Arclength

\section{Introduction}

Let $B$ denote the set of all analytic functions $a$ defined on the unit disk $U=\{z:|z|<1\}$ having the property that $|a(z)|<1$ for all $z \in U$. A logharmonic mapping defined on the unit disk $U$ is a solution of the nonlinear elliptic partial differential equation

$$
\frac{\overline{f_{\bar{z}}}}{\bar{f}}=a \frac{f_{z}}{f},
$$

where the second dilatation function $a \in B$. Because $a \in B$, the Jacobian 


$$
J_{f}=\left|\frac{f_{z}}{f}\right|^{2}\left(1-|a|^{2}\right)
$$

is positive and hence, non-constant logharmonic mappings are sense-preserving and open on $U$. If $f$ is a nonconstant logharmonic mapping of $U$ and vanishes at $z=0$ but has no other zeros in $U$, then $f$ admits the following representation

$$
f(z)=z^{m}|z|^{2 \beta m} h(z) \overline{g(z)}
$$

where $m$ is a nonnegative integer, $\Re(\beta)>-1 / 2$ and, $h$ and $g$ are analytic functions in $U$ with $g(0)=1$ and $h(0) \neq 0$ ([1]). The exponent $\beta$ in (1.2) depends only on $a(0)$ and can be expressed by

$$
\beta=\overline{a(0)} \frac{1+a(0)}{1-|a(0)|^{2}} .
$$

Note that $f(0) \neq 0$ if and only if $m=0$ and that a univalent logharmonic mapping on $U$ vanish at the origin if and only if $m=1$. Thus, a univalent logharmonic mappings on $U$ which vanishes at the origin will be of the form

$$
f(z)=z|z|^{2 \beta} h(z) \overline{g(z)},
$$

where $\Re(\beta)>-1 / 2$ and $0 \notin(h g)(U)$, and have been studied extensively in the recent years, see [1]-[7]. In this case, it follows that $F(\zeta)=\log f\left(\mathrm{e}^{\zeta}\right)$ are univalent harmonic mappings of the half-plane

$\{\zeta: \Re(\zeta)<0\}$, a detail study of univalent harmonic mappings to be found in [8]-[14]. Such mappings are closely related to the theory of minimal surfaces, see [15] [16].

Let $f=z|z|^{2 \beta} h \bar{g}$ be a univalent logharmonic mapping. We say that $f$ is starlike logharmonic mapping if

$$
\frac{\partial \arg f\left(r \mathrm{r}^{i \theta}\right)}{\partial \theta}=\mathfrak{R} \frac{z f_{z}-\bar{z} f_{\bar{z}}}{f}>0
$$

for all $z \in U$. Denote by $S T_{L h}^{*}$ the set of all starlike logharmonic mappings, and by $S^{*}$ the set of all starlike analytic mappings. It was shown in [4] that

$f(z)=z|z|^{2 \beta} h(z) \overline{g(z)} \in S T_{L h}^{*}$ if and only if $\psi(z)=z h(z) / g(z) \in S^{*}$.

It is rather a natural question to ask whether there exists a linkage between the starlikeness of $f(z)=z h(z) \overline{g(z)}$ and $\phi(z)=z h(z)$.

In Section 2, we determine the radius of starlikeness for the logharmonic mapping $f(z)=z h \bar{g}$ where $\varphi=z h \in S^{*}$. A distortion theorem and an upper bound for the arclength of these mappings will be included.

In Section 3, we discuss the integral means for logharmonic mappings associated to starlike analytic mappings.

\section{Basic Properties of Mappings from $S_{L h}^{*}$.}

We start this section by establishing a linkage between the starlikeness of $f(z)=z h \bar{g}$ and $\varphi(z)=z h(z)$.

Theorem 1 a) Let $f(z)=z h \bar{g}$ be a logharmonic mapping where $\varphi(z)=z h(z) \in S^{*}$. Then $f$ maps the disk $\left\{z:|z|<R_{0}\right\}$, where $R_{0}=0.2956$ onto a starlike domain.

b) If $f(z)=z h \bar{g} \in S T_{L h}^{*}$. Then $\varphi(z)=z h(z)$ maps the disk $\left\{z:|z|<R_{0}\right\}$, where $R_{0}=0.2956$ onto a starlike domain.

Proof. a) Let $f(z)=z h \bar{g}$ be a logharmonic mapping with respect to $a \in B$ and $a(0)=0$. Suppose that $\varphi(z)=z h(z) \in S^{*}$. Then $f(z)$ can be written in the form

$$
f(z)=\varphi(z) \exp \overline{\int_{0}^{z} a(s) \frac{\varphi^{\prime}(s)}{\varphi(s)} \mathrm{d} s .}
$$

A simple calculations leads to

$$
\frac{\partial \arg f(z)}{\partial \theta}=\mathfrak{R} \frac{z f_{z}-\bar{z} f_{\bar{z}}}{f}=\Re(1-a(z)) \frac{z \varphi^{\prime}(z)}{\varphi(z)}=\mathfrak{R} \frac{z \varphi^{\prime}(z)}{\varphi(z)}-\mathfrak{R}\left(a(z) \frac{z \varphi^{\prime}(z)}{\varphi(z)}\right),
$$


where $z=r \mathrm{e}^{i \theta}$. Since $\mathfrak{R} \frac{z \varphi^{\prime}(z)}{\varphi(z)} \geq \frac{1-r}{1+r}$ and $\mathfrak{R}\left(a(z) \frac{z \varphi^{\prime}(z)}{\varphi(z)}\right) \leq \frac{r(1+r)}{(1-r)}$,

we obtain

$$
\mathfrak{R} \frac{\mathrm{zf}_{z}-\overline{\mathrm{z}} f_{\overline{\mathrm{z}}}}{f} \geq \frac{1-r}{1+r}-\frac{r(1+r)}{(1-r)}
$$

This gives

$$
\Re \frac{z f_{z}-\bar{z} f_{\bar{z}}}{f} \geq \frac{1-3 r-r^{2}-r^{3}}{\left(1-r^{2}\right)} .
$$

Thus $\Re \frac{z f_{z}-\bar{z} f_{\bar{z}}}{f}>0$ if $1-3 r-r^{2}-r^{3}>0$. Therefore, the radius of starlikeness $\rho$ is the smallest positive root (less than 1 ) of $1-3 r-r^{2}-r^{3}=0$ which is $R_{0}=0.2956$. We conclude that $f$ is univalent in $\left\{z:|z|<R_{0}\right\}$ and maps the disk $\left\{z:|z|<R_{0}\right\}$ onto a starlike domain.

b) Let $f(z)=z h \bar{g}$ be a starlike logharmonic mapping defined on the unit disk $U$ with respect to $a \in B$ with $a(0)=0$. Then by [4] $\psi(z)=\frac{z h(z)}{g(z)} \in S^{*}$

and also,

$$
g(z)=\exp \int_{0}^{z} \frac{a(s)}{1-a(s)} \frac{\psi^{\prime}(s)}{\psi(s)} \mathrm{d} s
$$

Hence,

$$
\varphi(z)=z h(z)=\psi(z) g(z)
$$

and then simple calculations give that

$$
\begin{aligned}
\frac{\partial \arg \varphi(z)}{\partial \theta} & =\mathfrak{R} \frac{z \varphi^{\prime}(z)}{\varphi(z)}=\mathfrak{R}\left(\frac{1}{1-a(z)} \frac{z \psi^{\prime}(z)}{\psi(z)}\right)=\frac{1}{\mid 1-a(z)^{2}} \mathfrak{R}\left((1-\overline{a(z)}) \frac{z \psi^{\prime}(z)}{\psi(z)}\right) \\
& =\frac{1}{|1-a(z)|^{2}}\left[\mathfrak{R} \frac{z \varphi^{\prime}(z)}{\varphi(z)}-\mathfrak{R}\left(\overline{a(z)} \frac{z \varphi^{\prime}(z)}{\varphi(z)}\right)\right] \geq \frac{1}{(1+r)^{2}}\left[\frac{1-r}{1+r}-\frac{r(1+r)}{(1-r)}\right] \\
& =\frac{1}{(1+r)^{2}} \frac{1-3 r-r^{2}-r^{3}}{\left(1-r^{2}\right)} .
\end{aligned}
$$

Thus $\mathfrak{R} \frac{z \varphi^{\prime}(z)}{\varphi(z)}>0$ if $1-3 r-r^{2}-r^{3}>0$. Therefore, the radius of starlikeness $\rho$ is the smallest positive root (less than 1) of $1-3 r-r^{2}-r^{3}=0$ which is $R_{0}=0.2956$. We conclude that $\varphi$ is univalent in $\left\{z:|z|<R_{0}\right\}$ and maps the disk $\left\{z:|z|<R_{0}\right\}$ onto a starlike domain.

Our next result is a distortion theorem for the set of all logharmonic mappings $f(z)=z h \bar{g}$ where $\varphi(z)=z h(z) \in S^{*}$

Theorem 2 Let $f(z)=z h \bar{g}$ be a logharmonic mapping defined on the unit disk $U$ where $\varphi(z)=z h(z) \in S^{*}$, then for $z \in U$,

i) $\frac{|z|(1-|z|)^{2}}{(1+|z|)^{2}} \mathrm{e}^{|z|} \leq|f(z)| \leq \frac{|z|}{(1-|z|)^{4}} \mathrm{e}^{-|z|}$

ii) $\left|f_{z}(z)\right| \leq \frac{(1+|z|)}{(1-|z|)^{5}} \mathrm{e}^{-|z|}$ 
iii) $\left|f_{\bar{z}}(z)\right| \leq \frac{|z|(1+|z|)}{(1-|z|)^{5}} \mathrm{e}^{-|z|}$

Equality holds for the right hand side if and only if $a(z)=\eta z$ and $\varphi(z)=\frac{z}{(1-z)^{2}}$ which leads to $f(z)=\bar{\eta} f_{0}(\eta z)$, where

$$
f_{0}(z)=\frac{z}{|1-z|^{4}} \mathrm{e}^{-\bar{z}}
$$

Proof. i) Let $f(z)=z h \bar{g}$ be a logharmonic mapping with respect to $a \in B$ with $a(0)=0$. Suppose that $\varphi(z)=z h(z) \in S^{*}$. Then $f(z)$ can be written in the form

$$
f(z)=\varphi(z) \overline{\exp } \overline{\int_{0}^{z} a(s) \frac{\varphi^{\prime}(s)}{\varphi(s)} \mathrm{d} s .}
$$

For $|z|=r$, we have

$$
\frac{r}{(1+r)^{2}} \leq|\varphi(z)| \leq \frac{r}{(1-r)^{2}}
$$

and

$$
\left|a(z) \frac{\varphi^{\prime}(z)}{\varphi(z)}\right| \leq \frac{1+r}{1-r} .
$$

Combining (2.2), (2.3) and (2.4), we get

$$
|f(z)| \leq \frac{r}{(1-r)^{2}} \exp \Re \int_{0}^{r} \frac{1+t}{1-t} \mathrm{~d} t=\frac{r}{(1-r)^{4}} \mathrm{e}^{-r} .
$$

Equality holds for the right hand side if and only if $a(z)=\eta z$ and $\varphi(z)=\frac{z}{(1-z)^{2}}$ which leads to $f(z)=\bar{\eta} f_{0}(\eta z)$.

For the left hand side inequality, we have

$$
|f(z)|=|\varphi(z)| \exp \Re \int_{0}^{z} a(s) \frac{\varphi^{\prime}(s)}{\varphi(s)} \mathrm{d} s \geq \frac{r}{(1+r)^{2}} \exp -\int_{0}^{r} \frac{1+s}{1-s} \mathrm{~d} s=\frac{r(1-r)^{2}}{(1+r)^{2}} \mathrm{e}^{r} .
$$

ii) and iii) Differentiation $f(z)$ in (2.2) with respect to $z$ and $\bar{Z}$ respectively leads to

$$
f_{z}(z)=f(z) \frac{\varphi^{\prime}(z)}{\varphi(z)}
$$

and

$$
f_{\bar{z}}(z)=f(z) \frac{a(z) \varphi^{\prime}(z)}{\varphi(z)} .
$$

The result follows from substituting from Theorem 2(i), (2.3) and (2.4) into (2.5) and (2.6).

In the next theorem we establish an upper bound for the arclength of the set of all logharmonic mappings $f(z)=z h \bar{g}$ where $\varphi(z)=z h(z) \in S^{*}$

Theorem 3 Let $f(z)=z h \bar{g}$ be a logharmonic mapping defined on the unit disk $U$ where $\varphi(z)=z h(z) \in S^{*}$. Suppose that for $r, 0<r<1, \quad\left|f\left(r \mathrm{e}^{\mathrm{i} \theta}\right)\right| \leq M(r)$ then 


$$
L(r) \leq 4 \pi M(r) \frac{1+r}{1-r}
$$

Proof. Let $C_{r}$ denote the closed curve which is the image of the circle $|z|=r<1$ under the mapping $w=f(z)$. Then

$$
L(r)=\int_{C_{r}}|\mathrm{~d} f|=\int_{0}^{2 \pi}\left|z f_{\bar{z}}-\bar{z} f_{\bar{z}}\right| \mathrm{d} \theta=\int_{0}^{2 \pi}|f|\left|\frac{z f_{z}-\bar{z} f_{\bar{z}}}{f}\right| \mathrm{d} \theta \leq M(r) \int_{0}^{2 \pi}\left|\frac{z f_{z}-\bar{z} f_{\bar{z}}}{f}\right| \mathrm{d} \theta .
$$

Now using (2.5) and (2.6) we have

$$
\frac{z f_{z}-\bar{z} f_{\bar{z}}}{f}=\mathfrak{R}\left((1-a(z)) \frac{z \varphi^{\prime}(z)}{\varphi(z)}\right)+i \Im\left[(1+a(z)) \frac{z \varphi^{\prime}(z)}{\varphi(z)}\right] .
$$

Therefore,

$$
\begin{aligned}
L(r) & \leq M(r) \int_{0}^{2 \pi} \mathfrak{R}\left((1-a(z)) \frac{z \varphi^{\prime}(z)}{\varphi(z)}\right) \mathrm{d} \theta+M(r) \int_{0}^{2 \pi}\left|\mathfrak{J}\left[(1+a(z)) \frac{z \varphi^{\prime}(z)}{\varphi(z)}\right]\right| \mathrm{d} \theta \\
& =M(r) \cdot I_{1}+M(r) I_{2} .
\end{aligned}
$$

Since $\mathfrak{R}\left((1-a(z)) \frac{z \varphi^{\prime}(z)}{\varphi(z)}\right)$ is harmonic, and by the mean value theorem for harmonic functions, $I_{1}=2 \pi$. Also, $(1+a(z)) \frac{z \varphi^{\prime}(z)}{\varphi(z)}$ is subordinate to $\frac{(1+z)^{2}}{(1-z)}$,

therefore, we have

$$
I_{2}=\int_{0}^{2 \pi}\left|\mathfrak{J}\left[(1+a(z)) \frac{z \varphi^{\prime}(z)}{\varphi(z)}\right]\right| \mathrm{d} \theta \leq \int_{0}^{2 \pi}\left|\frac{(1+z)^{2}}{(1-z)}\right| \mathrm{d} \theta \leq 2 \pi \frac{(1+|z|)^{2}}{1-|z|} .
$$

Substituting the bounds for $I_{1}$ and $I_{2}$ in (2.8), we get

$$
\begin{aligned}
& L(r) \leq 2 \pi M(r)+2 \pi M(r) \frac{(1+r)^{2}}{(1-r)} \\
& L(r)=O(1) M(r) \frac{1}{1-r} .
\end{aligned}
$$

\section{Integral Means}

Theorem 4 of this section is an applications of the Baerstein star functions to the class of logharmonic mappings $f(z)=z h \bar{g}$ defined on the unit disk $U$ where $\varphi(z)=z h(z) \in S^{*}$. Star function was first introduced and properties were derived by Baerstein [17] [18], [Chapter 7]. The first application was the remarkable result, if $f \in S$ then

$$
\int_{-\pi}^{\pi}\left|f\left(r \mathrm{e}^{i t}\right)\right|^{p} \mathrm{~d} t \leq \int_{-\pi}^{\pi}\left|k\left(r \mathrm{e}^{i t}\right)\right|^{p} \mathrm{~d} t,
$$

where $k(z)=\frac{z}{(1-z)^{2}}, 0<r<1$ and $p>0$.

If $u(z)$ is a real $L^{1}$ function in an annulus $0<R_{1}<|z|<R_{2}$ then the definition of the star function of $u$, $u^{*}$ is

$$
u^{*}\left(r \mathrm{e}^{\mathrm{i} \theta}\right)=\sup _{|E|=2 \theta} \int_{E} u\left(r \mathrm{e}^{i t}\right) \mathrm{d} t \text {, for } R_{1}<r<R_{2} .
$$

One important property is that when $u$ is symmetric (even) re-arrangement then 


$$
u^{*}\left(r \mathrm{e}^{\mathrm{i} \theta}\right)=\int_{-\theta}^{\theta} u\left(r \mathrm{e}^{\mathrm{it}}\right) \mathrm{d} t .
$$

Other properties [18], [Chapter 7] are that the star-function is sub-additive and star respects subordination. Respect means that the star of the subordinate function is less than or equal to the star of the function. In addition, it was also shown that star-function is additive when functions are symmetric re-arrangements. Here is a lemma, quoted in [18], [Chapter 7] which we will use later.

Lemma 1 For $g, h$ real and $L^{1}$ on $[-a, a]$, the following are equivalent

a) For every convex non-decreasing function $\Phi: R \rightarrow R$

$$
\int_{-a}^{a} \Phi(g(x)) \mathrm{d} x \leq \int_{-a}^{a} \Phi(h(x)) \mathrm{d} x .
$$

b) For every $t \in R$,

$$
\int_{-a}^{a}(g(x)-t)^{+} \mathrm{d} t \leq \int_{-a}^{a}(h(x)-t)^{+} \mathrm{d} t .
$$

c) For every $x \in[0, a]$,

$$
g^{*}(x) \leq h^{*}(x) .
$$

Our main result of this section is the following theorem.

Theorem 4 If $f(z)=z h \bar{g}$ be a logharmonic mapping defined on the unit disk $U$ where $\varphi(z)=z h(z) \in S^{*}$ then for each fixed $r, \quad 0<r<1$ and as a function of $\theta$

$$
(\log |f(z)|)^{*} \leq\left[\log \left[\left|\frac{z}{(1-z)^{2}}\right| \exp \left(\int_{0}^{1} r\left|\frac{1+\rho r \mathrm{e}^{i t}}{1-\rho r \mathrm{e}^{i t}}\right| \mathrm{d} \rho\right)\right]\right]^{*} .
$$

Equality occurs if and only if $f_{\circ}(z)=z h_{\circ}(z) \overline{g_{\circ}(z)}$ is one of the functions of the form $\bar{\eta} f_{\circ}(\eta z),|\eta|=1$, where

$$
f_{\circ}(z)=\frac{z}{|1-z|^{4}} \exp (\Re(-z))
$$

Proof. Let $f(z)=z h(z) \overline{g(z)}=\varphi(z) \overline{g(z)} \in S_{L h}^{*}$, then by (2.2), we have

$$
f(z)=\phi(z) \exp \overline{\left(\int_{0}^{1} a(\rho z) \frac{z \phi^{\prime}(\rho z)}{\phi(\rho z)} \mathrm{d} \rho\right)},
$$

where $\phi(z)=S^{*}$ and $a \in B$.

Then

$$
\log |f(z)|=\log |\phi(z)|+\operatorname{Re}\left(\int_{0}^{1} a(\rho z) \frac{z \phi^{\prime}(\rho z)}{\phi(\rho z)} \mathrm{d} \rho\right) \text {, where } z=r \mathrm{e}^{i \theta} .
$$

Write $a(\rho z) \frac{z \phi^{\prime}(\rho z)}{\phi(\rho z)}=a(\rho z) \frac{1+\omega(\rho z)}{\rho(1-\omega(\rho z))}$, where $\omega$ is analytic, $|\omega|<1$ and $\omega(0)=0$, (see [9]).

As the star-function is sub-additive,

But since

$$
\begin{aligned}
(\log |f(z)|)^{*} & \leq(\log |\phi(z)|)^{*}+\int_{0}^{1}\left(\operatorname{Re}\left(a(\rho z) \frac{1+\omega(\rho z)}{\rho(1-\omega(\rho z))}\right)\right)^{*} \mathrm{~d} \rho \\
& \left.\leq(\log |\phi(z)|)^{*}+\int_{0}^{1}\left(\mid a(\rho z) \frac{1+\omega(\rho z)}{\rho(1-\omega(\rho z))}\right)\right)^{*} \mathrm{~d} \rho .
\end{aligned}
$$




$$
\log |a(\rho z)| \frac{1+w(\rho z)}{1-w(\rho z)}|=\log | a(\rho z)|+\log | \frac{1+w(\rho z)}{1-w(\rho z)} \mid,
$$

each is subharmonic. $\log |a(\rho z)|$ is subordinate to $\log |\rho z|$ and $\log \left|\frac{1+w(\rho z)}{1-w(\rho z)}\right|$ is subordinate to $\log \left|\frac{1+\rho z}{1-\rho z}\right|$. Hence

$$
\log |a(\rho z)|^{*} \leq \log |\rho r|^{*}=\log |\rho r|,
$$

and

$$
\log \left|\frac{1+w(\rho z)}{1-w(\rho z)}\right|^{*} \leq \log \left|\frac{1+\rho r \mathrm{e}^{i t}}{1-\rho r \mathrm{e}^{i t}}\right|^{*}
$$

Then,

$$
\begin{aligned}
& \left(\log |a(\rho z)|\left|\frac{1+w(\rho z)}{1-w(\rho z)}\right|\right)^{*} \leq \log |a(\rho z)|^{*}+\log \left|\frac{1+w(\rho z)}{1-w(\rho z)}\right|^{*} \\
& \leq \log |\rho r|+\log \left|\frac{1+\rho r \mathrm{e}^{i t}}{1-\rho r \mathrm{e}^{i t}}\right|^{*}=\left(\log \rho r\left|\frac{1+\rho r \mathrm{e}^{i t}}{1-\rho r \mathrm{e}^{i t}}\right|\right)^{*}
\end{aligned}
$$

Thus,

$$
\left(\exp \left(\log |a(\rho z)| \frac{1+w(\rho z)}{1-w(\rho z)} \mid\right)\right)^{*} \leq \exp \left(\log |a(\rho z)|\left|\frac{1+w(\rho z)}{1-w(\rho z)}\right|\right)^{*} \leq \exp \left(\log \rho r\left|\frac{1+\rho r \mathrm{e}^{i t}}{1-\rho r \mathrm{e}^{i t}}\right|\right)^{*} .
$$

It follows that

$$
\left(|a(\rho z)| \frac{1+w(\rho z)}{1-w(\rho z)} \mid\right)^{*} \leq\left(\rho r\left|\frac{1+\rho r \mathrm{e}^{i t}}{1-\rho r \mathrm{e}^{i t}}\right|\right)^{*} .
$$

Consequently, by combining (3.4), (3.5) and using the fact that star-functions respect subordination, it follows that

$$
(\log |f(z)|)^{*} \leq\left(\log \left|\frac{z}{(1-z)^{2}}\right|\right)^{*}+\int_{0}^{1}\left(r\left|\frac{1+\rho r e^{i t}}{1-\rho r e^{i t}}\right|\right)^{*} \mathrm{~d} \rho .
$$

Hence, as star-functions are additive when functions are symmetric re-arrangements,

$$
\begin{aligned}
(\log |f(z)|)^{*} & \left.\leq\left(\log \left|\frac{z}{(1-z)^{2}}\right|\right)^{*}+\int_{0}^{1}\left(r\left|\frac{1+\rho r \mathrm{e}^{i t}}{1-\rho r \mathrm{e}^{i t}}\right|\right)\right)^{*} \mathrm{~d} \rho \\
& =\left[\log \left|\frac{z}{(1-z)^{2}}\right|+\int_{0}^{1} r \mid \frac{1+\rho r \mathrm{e}^{i t}}{1-\rho r \mathrm{e}^{i t} \mid} \mathrm{d} \rho\right]^{*} \\
& =\left[\log \left[\left|\frac{z}{(1-z)^{2}}\right| \exp \left(\int_{0}^{1} r\left|\frac{1+\rho r \mathrm{e}^{i t}}{1-\rho r \mathrm{e}^{i t}}\right| \mathrm{d} \rho\right)\right]\right]^{*} .
\end{aligned}
$$

Now by using Theorem 4 we have 
Corollary 1 If $f(z)=z h \bar{g}$ be a logharmonic mapping defined on the unit disk $U$ where $\varphi(z)=z h(z) \in S^{*}$ then $z=r e^{i \theta}$

$$
\int_{-\pi}^{\pi}|f(z)|^{p} \mathrm{~d} \theta \leq \int_{-\pi}^{\pi}|| \frac{z}{(1-z)^{2}}\left|\exp \left(\int_{0}^{1} r\left|\frac{1+\rho r \mathrm{e}^{i t}}{1-\rho r \mathrm{e}^{i t}}\right| \mathrm{d} \rho\right)\right|^{p} \mathrm{~d} \theta, \quad p>0
$$

and

$$
\int_{-\pi}^{\pi}\left(\log ^{+}|f(z)|\right) \mathrm{d} \theta \leq \int_{-\pi}^{\pi} \log ^{+}\left|\frac{z}{(1-z)^{2}}\right| \mathrm{d} \theta+\int_{-\pi}^{\pi} \Re\left(\int_{0}^{1} r\left|\frac{1+\rho r \mathrm{e}^{i t}}{1-\rho r \mathrm{e}^{i t}}\right| \mathrm{d} \rho\right) \mathrm{d} \theta \leq C<\infty,
$$

the later implies that $f \in N^{+}$, hence $f$ has radial limits.

Proof. Let $\Phi(x)=\exp (p x)$, this is non-decreasing convex function. The first integral mean can be obtained using part (a) of Lemma 1 and Theorem 4. Moreover, the choice $\Phi(x)=\log ^{+}(x)$ yields the second integral mean.

\section{References}

[1] Abdulhadi, Z. (1996) Close-to-Starlike Logharmonic Mappings. The International Journal of Mathematics and Mathematical Sciences, 19, 563-574.

[2] Abdulhadi, Z. (2002) Typically Real Logharmonic Mappings. The International Journal of Mathematics and Mathematical Sciences, 31, 1-9.

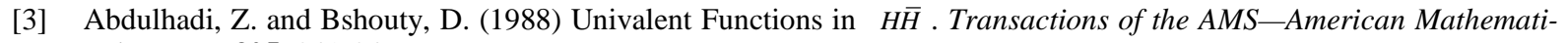
cal Society, 305, 841-849.

[4] Abdulhadi, Z. and Hengartner, W. (1987) Spirallike Logharmonic Mappings. Complex Variables, Theory and Application, 9, 121-130.

[5] Abdulhadi, Z., Hengartner, W. and Szynal, J. (1993) Univalent Logharmonic Ring Mappings. Proceedings of the American Mathematical Society, 119, 735-745.

[6] Abdulhadi, Z. and Hengartner, W. (1996) One Pointed Univalent Logharmonic Mappings. Journal of Mathematical Analysis and Applications, 203, 333-351.

[7] Abdulhadi, Z. and Hengartner, W. (2001) Polynomials in $H \bar{H}$. Complex Variables, Theory and Application, 46, 89107.

[8] Abu-Muhanna, Y. and Lyzzaik, A. (1990) The Boundary Behaviour of Harmonic Univalent Maps. Pacific Journal of Mathematics, 141, 1-20. http://dx.doi.org/10.2140/pjm.1990.141.1

[9] Clunie, J. and Sheil-Smal, T. (1984) Harmonic Univalent Functions. Annales Academiæe Scientiarum Fennicæe Mathematica, 9, 3-25.

[10] Duren, P. and Schober, G. (1987) A Variational Method for Harmonic Mappings on Convex Regions. Complex Variables, Theory and Application, 9, 153-168. http://dx.doi.org/10.1080/17476938708814259

[11] Duren, P. and Schober, G. (1989) Linear Extremal Problems for Harmonic Mappings of the Disk. Proceedings of the American Mathematical Society, 106, 967-973. http://dx.doi.org/10.1090/S0002-9939-1989-0953740-5

[12] Hengartner, W. and Schober, G. (1986) On the Boundary Behavior of Orientation-Preserving Harmonic Mappings. Complex Variables, Theory and Application, 5, 197-208. http://dx.doi.org/10.1080/17476938608814140

[13] Hengartner, W. and Schober, G. (1986) Harmonic Mappings with Given Dilatation. Journal London Mathematical Society, 33, 473-483. http://dx.doi.org/10.1112/jlms/s2-33.3.473

[14] Jun, S.H. (1993) Univalent Harmonic Mappings on $\Delta=\{z:|z|>1\}$. Proceedings of the American Mathematical Society, 119, 109-114. http://dx.doi.org/10.1090/S0002-9939-1993-1148026-3

[15] Nitsche, J.C.C. (1989) Lectures on Minimal Surfaces. Vol. 1, Translated from the German by Jerry M. Feinberg, Cambridge University Press, Cambridge.

[16] Osserman, R. (1986) A Survey of Minimal Surfaces. 2nd Edition, Dover, New York.

[17] Baernstein, A. (1974) Integral Means, Univalent Functions and Circular Symmetrization. Acta Mathematica, 133, 139169. http://dx.doi.org/10.1007/BF02392144

[18] Duren, P. (1983) Univalent Functions. Springer-Verlag, Berlin. 